\title{
ULTRAVIOLET PHOTOGRAPHY AND SPECTROSCOPY USING A SPECTRALLY SELECTIVÉ IMAGE CONVERTER
}

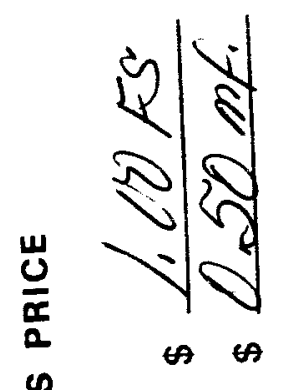

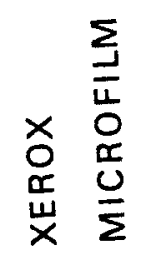

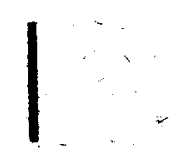

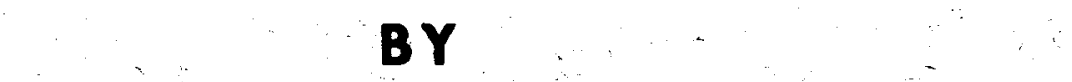

LAWRENCE DUNKELMAN JOHN HENNES
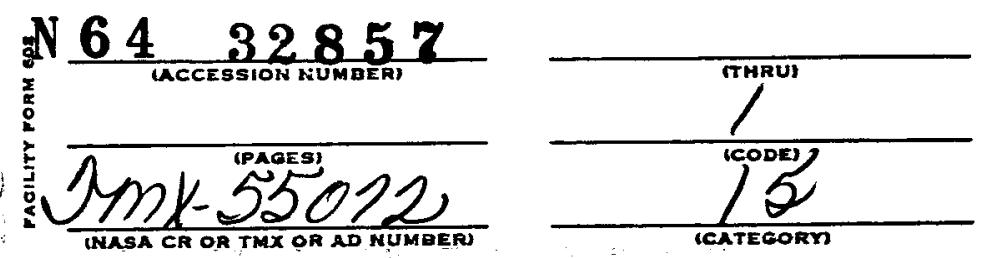
SEPTEMBER 1964

\section{NASA}

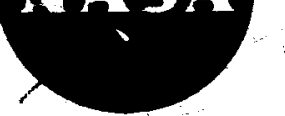

1
GODDARD SPACE FLIGHT CENTER GREENBELT, MARYLAND

Presented at the ICO Conference on Photographic and Spectroscopic Optics

Tokyo and Kyoto, Japan, September 1 - 8, 1964 To be published in a Supplement to the Japanese J. Applied Physics; May 1965 
ULTRAVIOLET PHOTOGRAPHY AND SPECTROSCOPY USING A SPECTRALLY SELECTIVE IMAGE CONVERTER

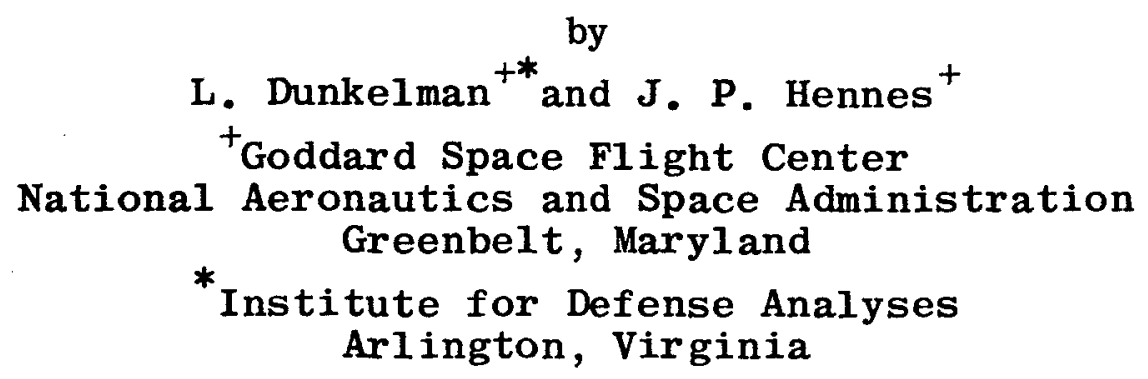

ABSTRACT

Laboratory and space research have both benefited from the new spectrally selective ultraviolet photodetectors. Large band-gap photoemitters, such as cesium telluride, having high quantum efficiencies in the ultraviolet and very low yields at longer wavelengths are now available. An image tube with such a cathode combines the advantages of spectrally selective photoemission with the flux-integrating property of photographic emulsion. Observations can thus be made at selected ultraviolet wavelength regions, either photographically or visually, even in the presence of strong long wavelength backgrounds. Visual measurements with a Cs-Te image converter, having a sapphire faceplate and a green phosphor, were made with the following results: (a) cathode quantum efficiency at $2537 \mathrm{~A}$ is 0.016 , which is expected to improve in future tubes; (b) minimal detectable converted 2537 A irradiance is $4 \times 10^{-5}$ ergs $\mathrm{cm}^{-2} \mathrm{sec}^{-1}$; and (c) tube background is undetectable. Mercury spectra obtained with this tube demonstrate the expected high rejection of visible light. Tables are included which 1 ist the brightnesses of several extended ultraviolet and visible sources of interest in the space sciences. The luminance of visible sources; the radiance of ultraviolet sources; and the relative brightness, on a photon basis, of spectrally converted ultraviolet sources along with visible sources are tabulated. 
There is a continuing need in spectroscopy and photography, in both laboratory and space research, for spectrally selective ultraviolet photodetection technicues. Photomultiplier detectors containing large band-sap photoemitting cathodes now exist wich effectively disciminate against long wavelengtis. (1) (2)

such a cathode can combine the advantages of spectially selective photoemission with either the flux-integrating property of photographic emulsion or the synthesizing ability of a human observer. This paper includes a brief discussion of some of the considerations to be given to the choice of window and photocathode materiais, extent of image intensification, etc. in converter design. Typical examples are given of the kinds of extended UV sources of astrophysical or geophysical origin which an astronaut might observe and photograph in various ultraviolet regions.

\section{INAGE TUBE CONSIDERATIONS}

McGee ${ }^{(3)}$ and Morton ${ }^{(4)}$ have recentiy provided excellent surveys of image tube development; accordingly, here we will include discussions of only chose image tube parameters unique to ultraviolet detection.

Figure 1 shows spectral response curves of some of the photocathodes used for ultraviolet photodetection. These emissive materials have been used in phototubes with appropriate ultraviolet transmitting faceplates such as LiF, $\mathrm{CaF}_{2}$, sapphire, fused silica, etc. The conventional Cs-Sb photoemitter is sensitive to visible light and is frequently not useful as an ultraviolet detector because of the presence of long wavelength straylight. The speciially selective cathodes $\mathrm{Cs}-\mathrm{Te}$ and $\mathrm{Cs}-\mathrm{I}$ are botin relatively insensitive to longer wavelengths and make excellent UV 
detectors. Metal photocathodes, such as tungsten, are insensitive in the visible and have low yield in the middle ultraviolet (3000-2000A), but reach about 10\% quantum yield at 1000A. Also shown is the relative quantum yield of Eastman 103a-0 spectroscopic film adapted from the Kodak handbook. (5) The response curves show that if a veak ultraviolet source must be observed in the presence of a strong visible background, then one must employ either spectral filtering or one of the spectrally selective cathodes, and occasionally both.

Figure 2 shows a variety of available ultraviolet transmitting windows whose short wavelength cutoffs range from $\lambda \lambda 1050 \mathrm{~A}$ (lithium fluoride) to $\lambda \lambda 3000 \mathrm{~A}$ (Corning $0-54$, glass). In general, these windows may be employed as the actual image tube faceplate. However, in practice it is usually easier to employ as the faceplate either an alkali halide, sapphire, fused silica or vycor material and provide for additional short wavelength cutofis by using either the appropriate material shown in Figure 2 or some other crystal, glass, gas cell, predisperser, etc. as desired by the user.

In this paper, we report on an ITT ultraviolet image tube with a cesium-telluride cathode and a sapphire faceplate. However, in Figure 3 there is shown a few of many other combinutions which are practicable, the spectral sensitivity curves being formed by combining the cathodes of Figure 1 with the windows of Figure 2. Note the relatively narrow spectral response of Cs-I with sapphire window; this region of $\lambda \lambda 1400$ to $2000 \mathrm{~A}$ might be of interest in the study of the Schumann-Runge $\mathrm{O}_{2}$ bands in the upper atmosphere. Note also the $\mathrm{K}-\mathrm{Br}$ cathode with LiF window yielding a response from $\lambda \lambda 1050$ to $1500 \mathrm{~A}$ for observing, for example, stellar or nebular fluxes. In many cases one 
would 'surely wish to exclude the strong Lyman $\alpha$ hydrogen emission "In" the upper atmosphere; here one could employ a $\mathrm{CaF}_{2}$ window or filter.

Figure 4 indicates three of many types of imaging systems: The top sketch, for example, shows a system which"might be used by an astronaut for making both photographic and visual surveys of geophysical or astronomical objects such as airglow, nebulosities, stellar and planetary sources, earth albedo, etc.(6)-(II) In this case, the image tube acts as a spectral filter and a wavelength converter. Conventional photographic film would be used rather than fragile ultraviolet sensitive spectroscopic films. The middle sketch demonstrates an objective grating spectrograph to observe and obtain, for example, stellar or planetary spectra; whereas the bottom sketch would be a useful scheme for observing a spectral energy distribution of airglow, nebulosities and aurorae. Since so little is known of the dynamic properties of ultraviolet aurorae, a movie camera might be substituted for the still camera depending on whether one were interested in maximizing the observations of spatial or spectral distributions. Additional discussion on the expected radiances of celestial objects is given in a later section.

The spectral selectivity of an ultraviolet image tube having a cesium telluride photocathode, is demonstrated by the photographs in Figure 5 . A tube manufactured by the ITT Industrial Laboratories in Fort Wayne, Indiana has been compared with a standard 7404 image tube with an S-13 cathode. Both are electrostatic, monovoltage tubes with identical imaging structures. The tubes were used to photograph a mercury arc spectrum in an arrangement similar to that illustrated in Figure 4 (bottom). The photographs show that although both tubes are equally sensitive to 
ultraviolet light, the Cs-Te ultraviolet tube is not affected at all by visible lines or stray light.

Figure 6 is a photograph of a display of electrooptical binoculars and the image tubes described in this paper. The upper right hand corner shows a pair of infrared binoculars which use standard infrared image tubes with cesium-silver-oxygen photocathodes. The image tubes shown are from left to right an RCA 7404 (Cs-Sb cathode with vycor window); an ITT UV tube (Cs-Te cathode with sapphire window); and an RCA far-UV tube (Cs-I cathode with LiF window). The image tube binocular, shown on the left with oculars and rubber shields removed, is equipped with quartz-CaF 2 doublet objective lenses with a $10.8 \mathrm{~cm}$ focal length shown in the detached barrels. The image tubes, housed in the central cylinders of the main body of the binocular, are powered by a 12 kilovolt miniature power supply. Monoculars rather than binoculars would be used in manned space observations since extraterrestrial observations would not appreciably benefit by double vision, certainly stereopsis is not involved here. Future developments of ultraviolet image tubes can include better collection optics and more sophisticated features such as multi-stage image intensification, magnetic focussing, simultaneous electronic readout, color translation, etc. One or more additional stages of image intensification (over the usual 30 or 40 gain in these single stage tubes) will be necessary in the cases of some of the interesting dim phenomena discussed in the next section.

The quantum efficiency of the photocatiode of the Cs-Te tube reported here is 0.016 . In 1958, when the first rubidium or cesium telluride photomultipliers were assembled the quantum efficiencies were even lower. 
Currently produced UV photomultipliers have yields of 0.05 or better and it is expected that this will be achieved with the image converters. Using our present tube, which has a green phosphor, as a converter we found that the minimal 2537A irradiance detectable by the eye was $4 \times 10^{-5}$ ergs $\mathrm{cm}^{-2} \mathrm{sec}^{-1}$. With the $\mathrm{f} / 5$ objective the minimal detectable 2537A radiance of an extended source was $0.01 \mathrm{erg} \mathrm{cm}^{-2} \mathrm{sec}^{-1} \mathrm{ster}^{-1}$. With this tube, the non-irradiated portion of the phosphor (the background) was black. This is what would be expected from large band-gap photoemitters which have negligible thermionic emission.

\section{THE ULTRAVIOLET SCENE FROM SPACE}

With reference to Table $I$, one can note the large variety and intensity range of extended (rather than point) source celestial objects of interest. These can be observed only from a vantage point above the ozonosphere (which absorbs the entire middle ultraviolet $\lambda 2000-3000 \mathrm{~A}$ ); or better still, at or above manned space altitudes ( 160 $\mathrm{Km}$ or more) to minimize absorption by oxygen. The table is divided into 3 sections, the first relates to phenomena in the visible region and is intended to provide (1) a convenient reference to objects fairly well known and whose observations are within our normal experience and (2) a means with which one can compare ultraviolet with visible sources. In the first section the luminance (brightness) of each source is given in $\mathrm{cd} \mathrm{cm}^{-2}$. In the second section of the table, there is listed again a wide variety of celestial UV sources all of which have been measured from above the ozonosphere. Here the radiance (brightness) is given in ergs $\mathrm{cm}^{-2} \mathrm{sec}^{-1} \mathrm{ster}^{-1}$. Finally, in the third section, some of these sources are listed relatively on a brightness scale. The ultraviolet fluxes have been converted to the corresponding 5500A fluxes on a one-for-one photon basis. 


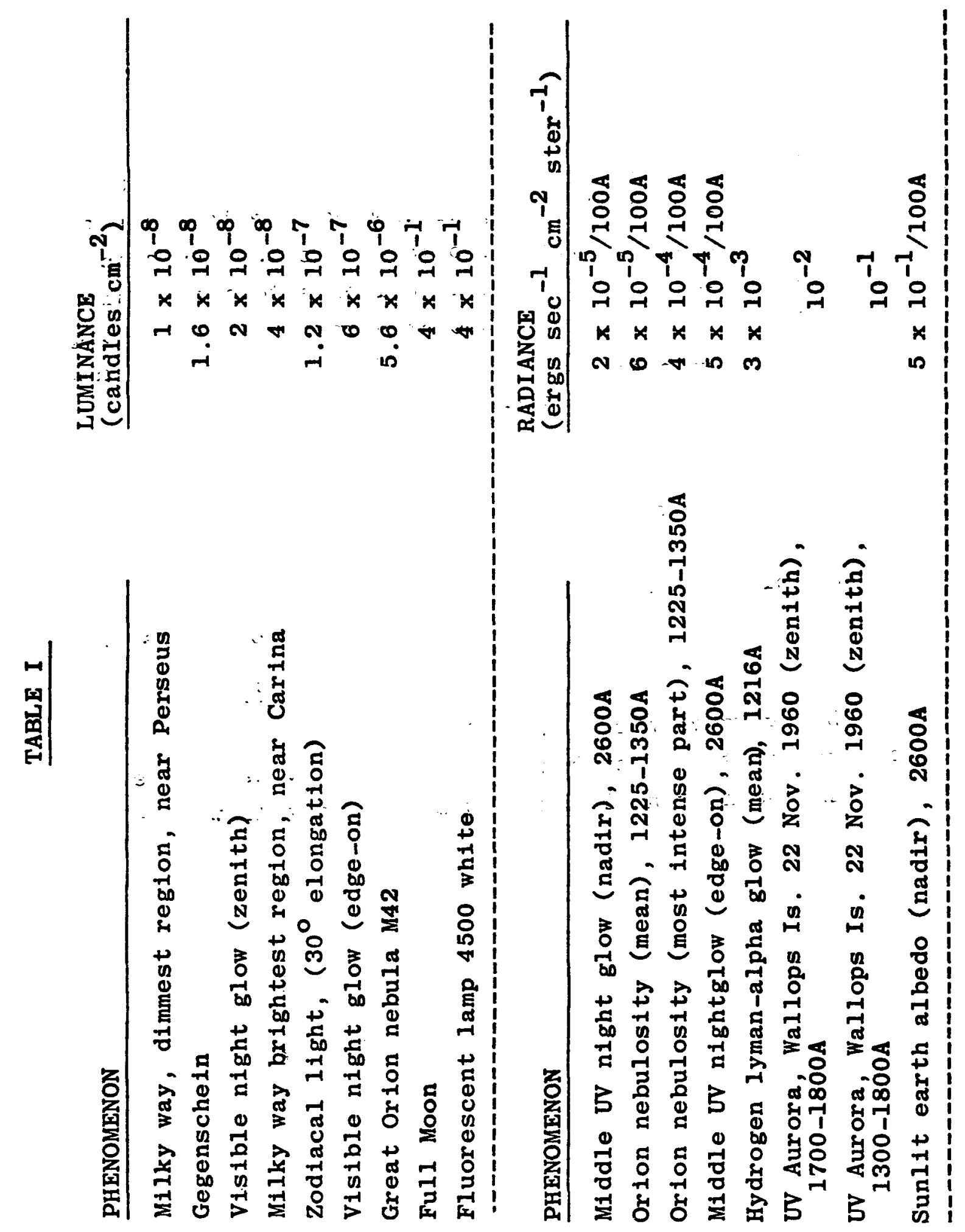


With fast collection optics and higher cathode efficiencies, ultraviolet sources of radiance as low as $10^{-4}$ ergs $\mathrm{cm}^{-2} \mathrm{sec}^{-1}$ ster $^{-1}$ should be visually detectable with only single stage image converters. This radiance level includes most of the sources listed in Table $I$. Less bright sources will require further amplification stages to be detectable. High amplification of very dim sources will not necessarily increase the information content for visual detection since photon statistical fluctuations at low flux levels limit image recognition (4). Intensification of moderate brightness sources will, of course, make visual observation more comfortable. Intensification of both weak and strong sources will reduce exposure times for photographic recording. This latter effect can be an important consideration in rocket as well as in manned space observations. 


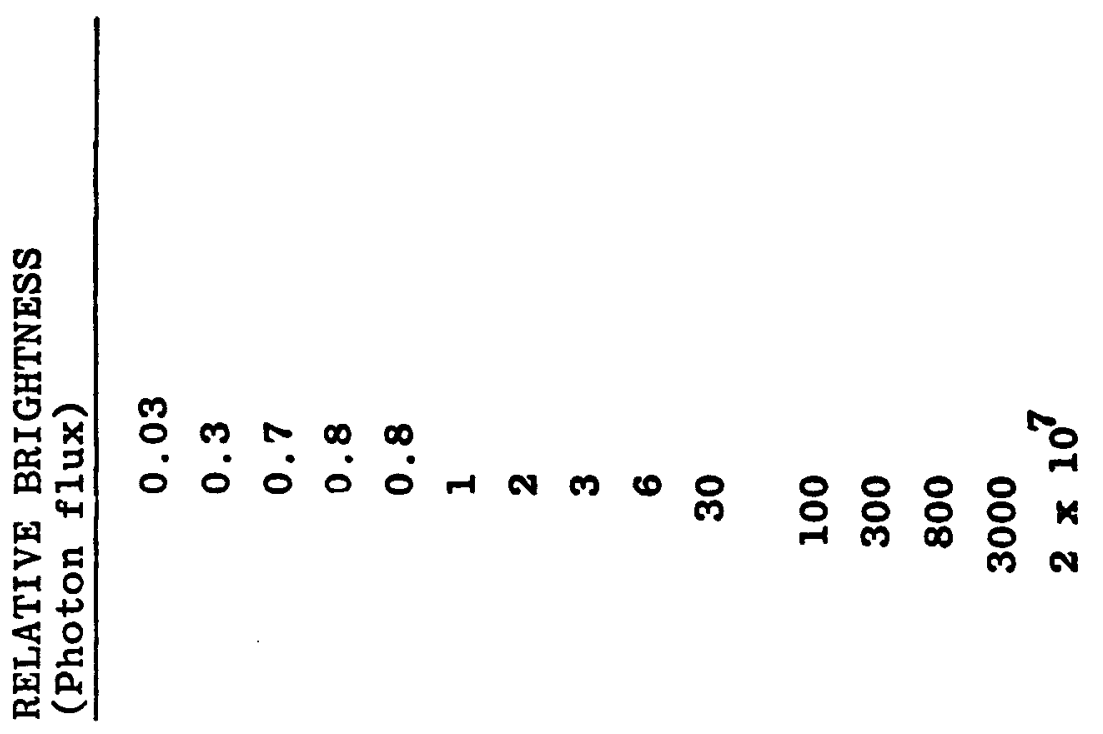

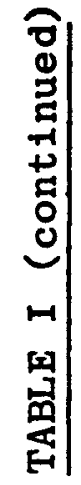

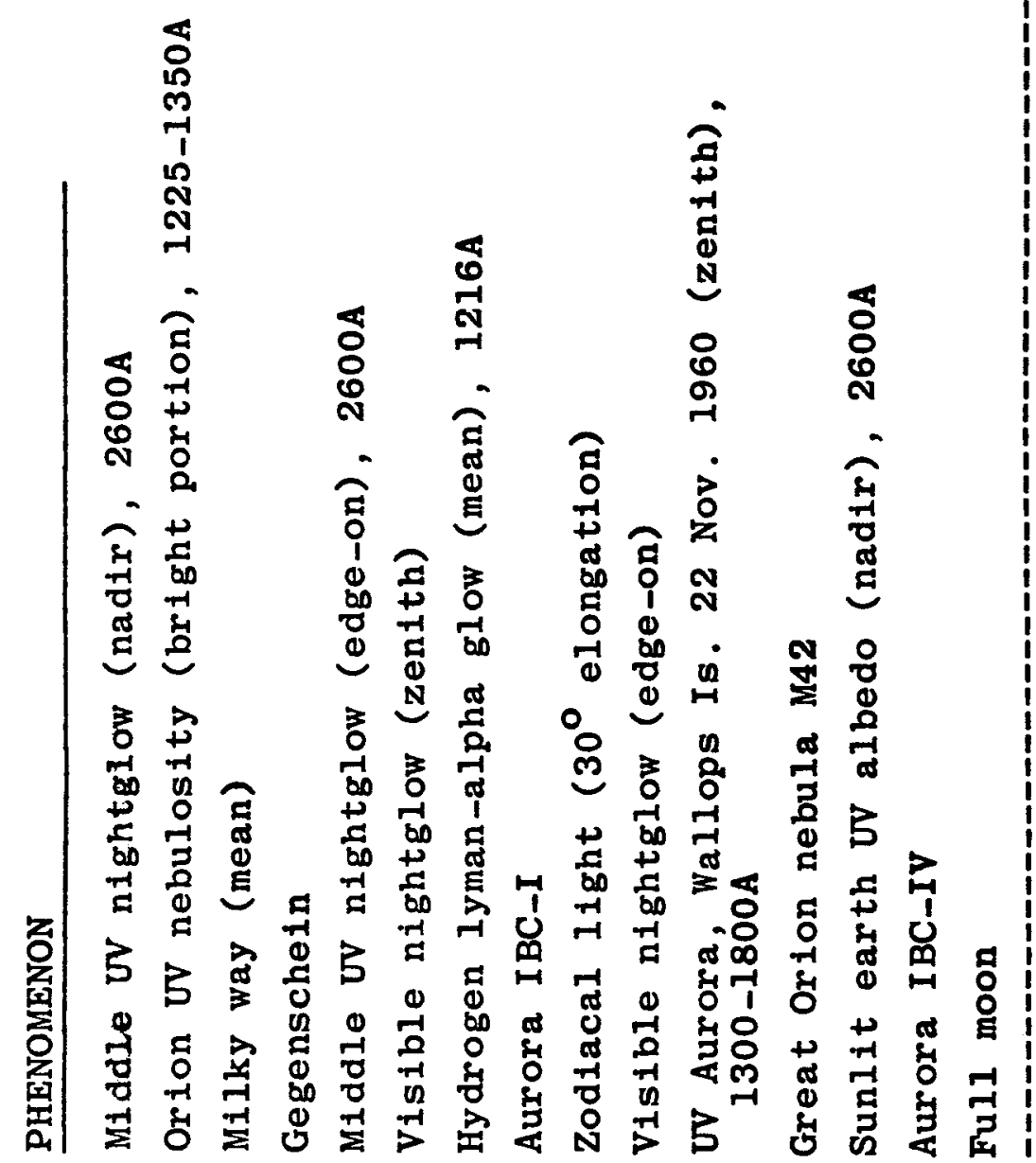




\section{REFERENCES}

1. L. Dunkelman, W.B. Fowler, and J. P. Hennes: Appl. Optics 1 (1962) 695.

2. L. Dunkelman: J. Quant. Spectrosc. Rad. Transfer 2 (1962) 533 .

3. J. D. McGee: Chapter 4.1, pp 81-103, in Space Age Astronomy (Academic Press, New York, 1962).

4. G. S. Morton: Appl. Optics 3 (1964) 651 .

5. Kodak Plates and Films for Science and Industry, (Eastman Kodak Co., Rochester, New York, 1962).

6. J. A. O'Keefe, L. Dunkelman, S. D. Soules, W. F. Huch, and P. D. Lowman, Jr.: Chapter 19, pp 327347, in Mercury Project Summary, NASA SP-45 (U.S. Govt. Print. Off., Washington, D.C. 1963).

7. L. Dunkelman, A. Boggess III, and R. Scolnik: Private communication.

8. J. E. Kupperian, Jr., A. Boggess III, and J. E. Milligan: Astrophys. J. 128 (1958) 453.

9. T. P. Stecher and J. E. Milligan: pp 1061-67 in Space Research III (North-Holland Publ. Co., Ams ter dam, 1963).

10. A. Boggess III: Chapter 7 in Space Astrophysics (McGraw-Hill, New York, 1961).

11. J. P. Hennes, W. B. Fowler, and L. Dunkelman: J. Geophys. Res. 69 (1964) 2835. 


\section{FIGURE CAPTIONS}

Figure 1: Spectral Response of Typical UV Photocathodes. These curves are taken from reference 1. The relative response of type 103a-0 film is from reference 5 .

Figure 2: UV Optical Materials.

Transmittance curves, uncorrected for surface reflections, are shown for materials of thickness: $1-1.2 \mathrm{~mm} ; 2-1 \mathrm{~mm} ; 3-1 \mathrm{~mm} ; 4-1 \mathrm{~mm} ; 5-2.5 \mathrm{~mm} ; 6-3$ $\mathrm{mm} ; 7,8$, and 9 are each about $2 \mathrm{~mm}$.

Figure 3: Spectrally Selective UV Photodetectors.

These curves are produced by combining some of the cathodes and filters from Figures 1 and 2 .

Figure 4: UV Imaging Systems.

Various possible optical systems for use with an image converter are shown. Either visual or photographic observation are represented in the top picture.

Figure 5: Hg Spectra With A UV Image Tube.

An RCA 7404 image tube ( $\mathrm{Cs}-\mathrm{Sb}$ cathode, vycor window) is on the left and an ITT UV Tube (Cs-Te cathode, sapphire window) is on the right. The UV tube has a slightly smaller cathode area.

A. The spectrum of the Hg lamp is shown on both tubes. The extreme left line is $3650 \mathrm{~A}$, the extreme right line is the green 5461A. Next to the first order green line is the second order 2537A line. Between the 2537A and the first order 3650A line are various blue and ultraviolet lines. Note that with the UV tube only the 2537A line and a very faint $3650 \mathrm{~A}$ trace are recorded. The visible and near UV lines do not appear at all. 
Figure 5: B. The spectrum of a $\mathrm{Hg}$ lamp in the presence of a strong continuum background (tungsten lamp) are recorded on both tubes. The visible image converter spectrum is almost washed out while the UV tube spectrum is the same as in part $A$.

Figure 6: UV Image Tubes and Binoculars. (See text) 


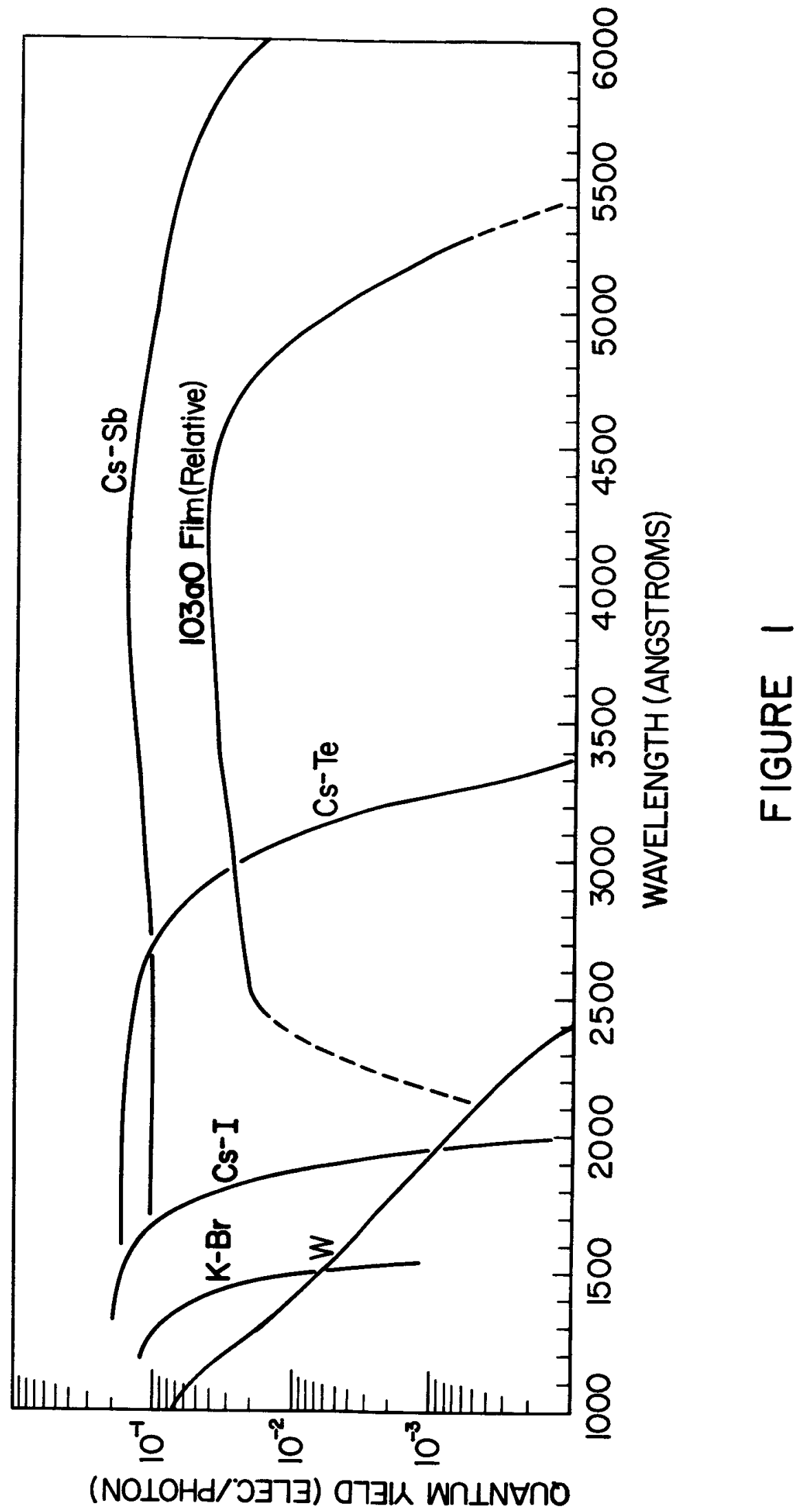




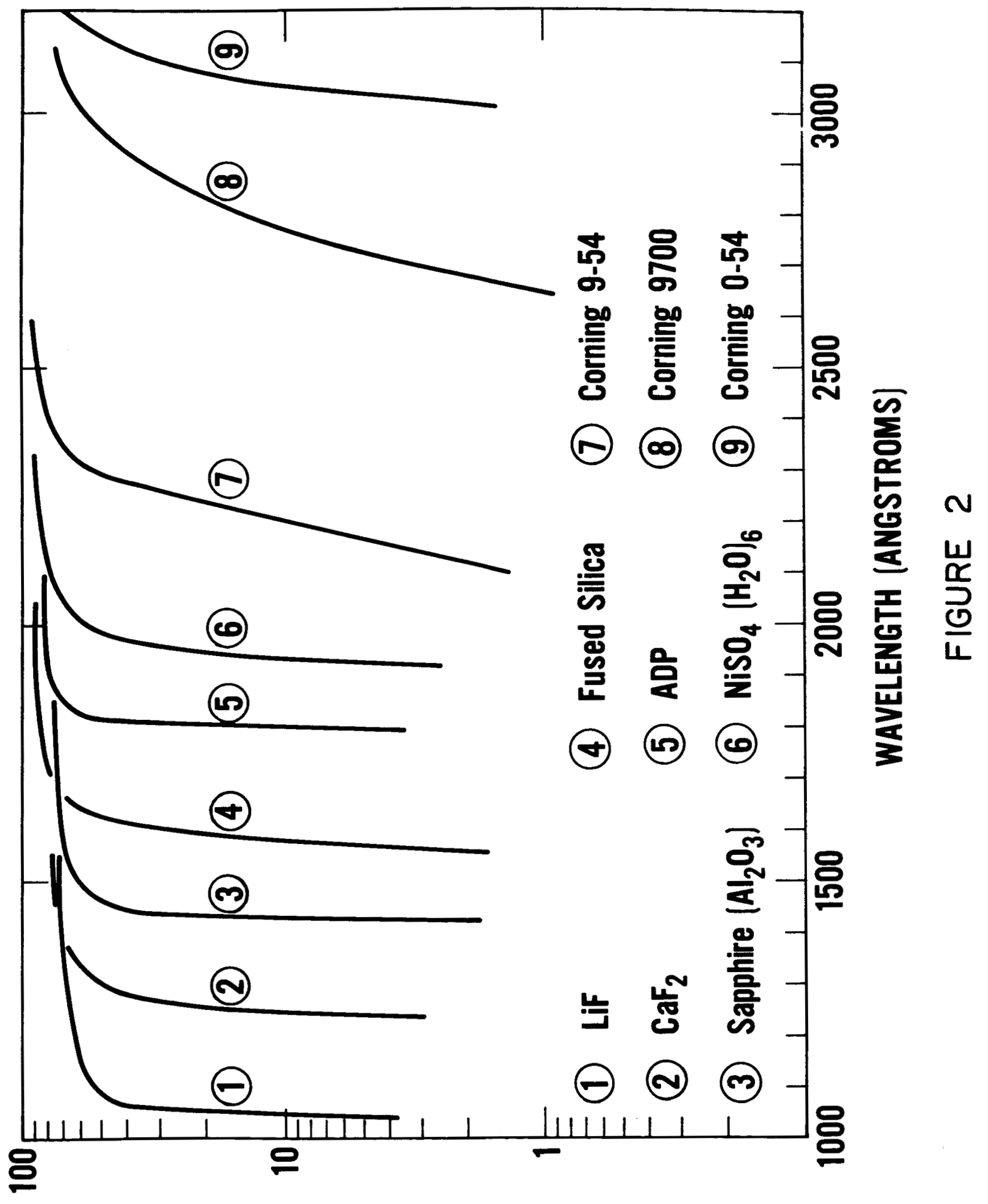

JJNYHIWSNYYL INJJYJd 


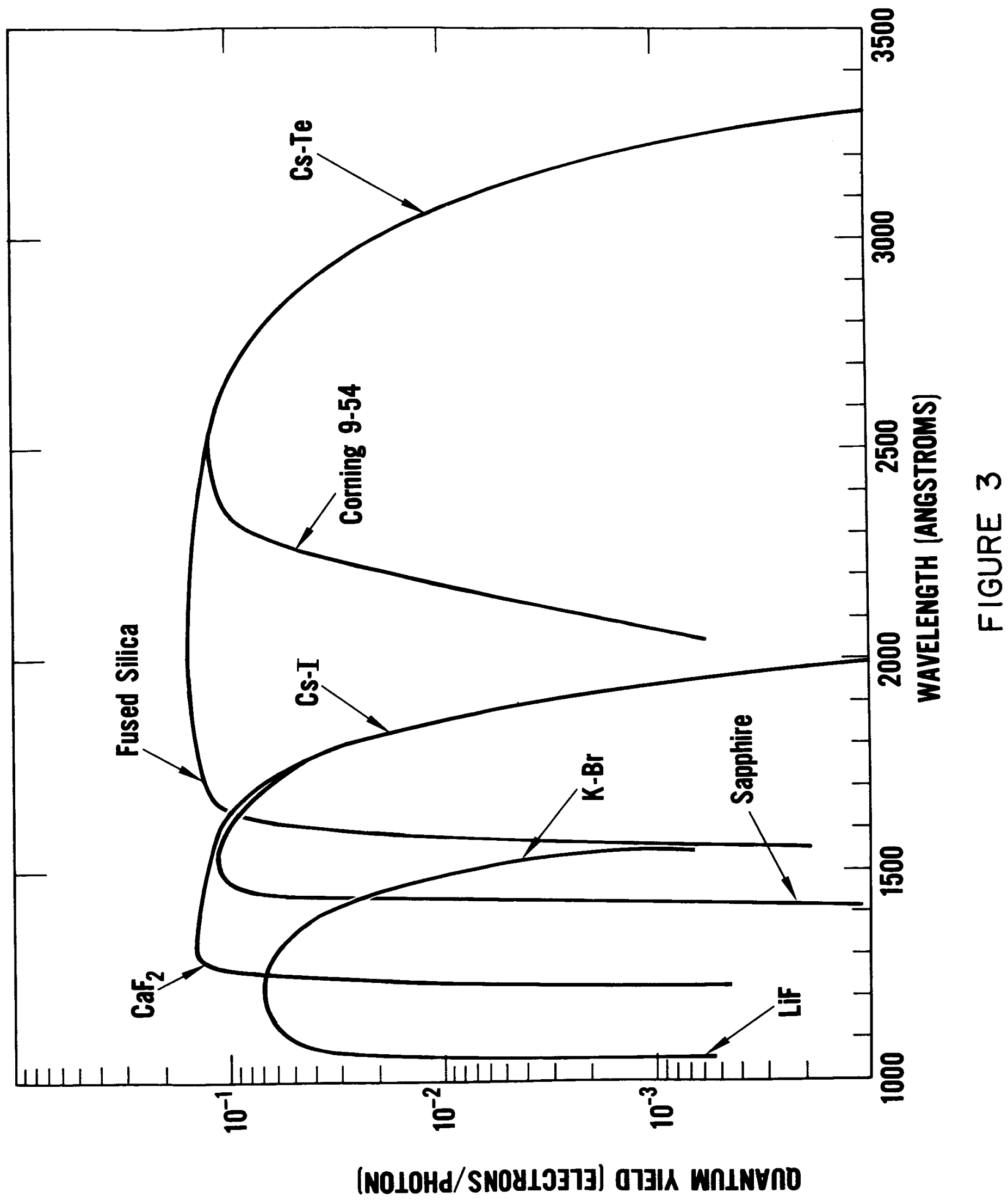




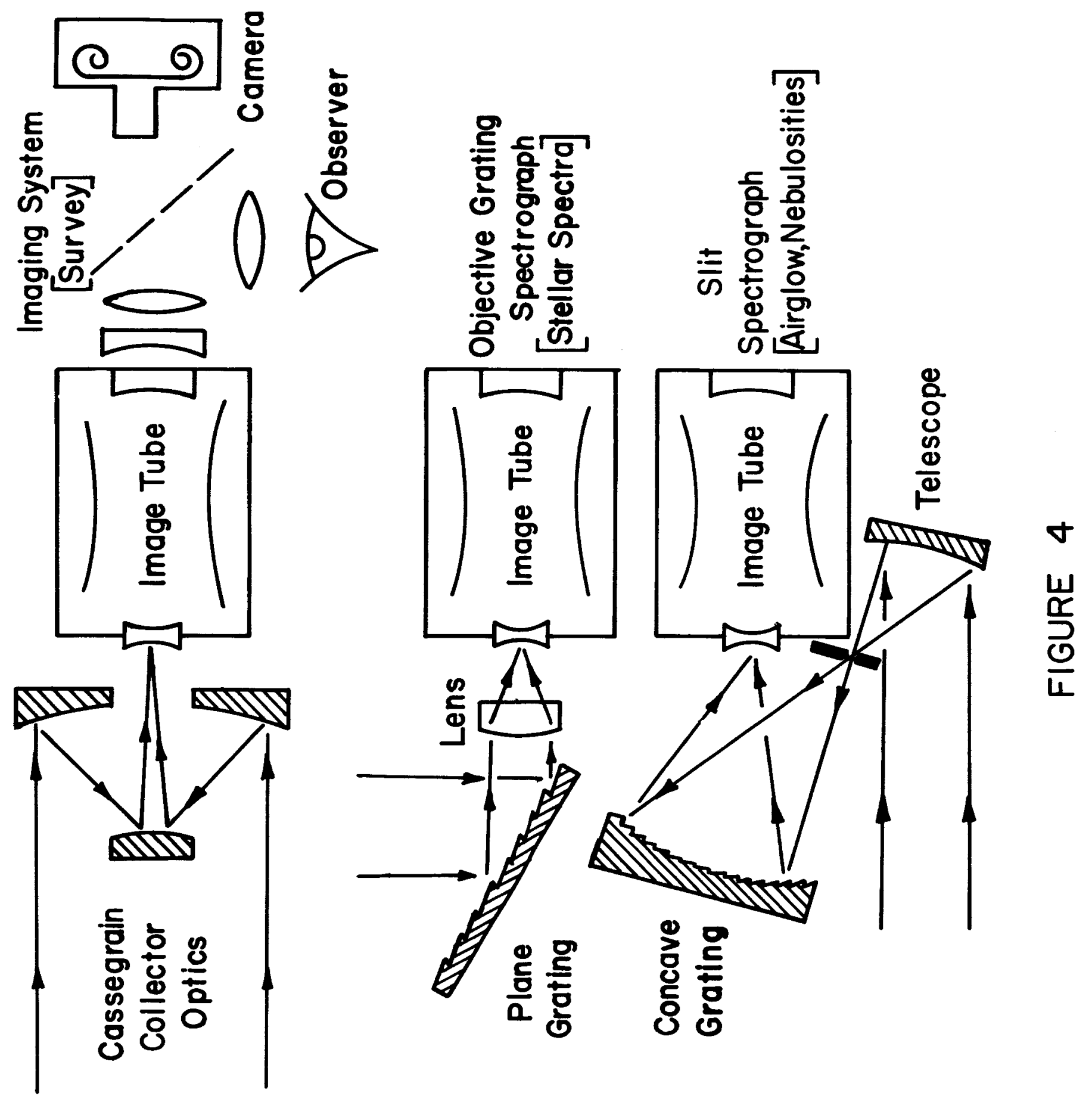




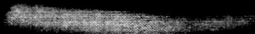

0
0
$\frac{1}{0}$
$\frac{0}{4}$ 
